\title{
Randomized study comparing 3D virtual reality and conventional 2D on-screen teaching of cerebrovascular anatomy
}

\author{
*Ladina Greuter, MD, ${ }^{1}$ Adriana De Rosa, ${ }^{2}$ Philippe Cattin, PhD, ${ }^{3}$ Davide Marco Croci, MD, ${ }^{1,4}$ \\ Jehuda Soleman, MD, ${ }^{1,2}$ and Raphael Guzman, MD'-3
}

${ }^{1}$ Department of Neurosurgery, University Hospital of Basel; 'Faculty of Medicine and ${ }^{3}$ Department of Biomedical Engineering, University of Basel, Switzerland; and ${ }^{4}$ Department of Neurosurgery, Clinical Neurosciences Center, University of Utah, Salt Lake City, Utah

\begin{abstract}
OBJECTIVE Performing aneurysmal clipping requires years of training to successfully understand the 3D neurovascular anatomy. This training has traditionally been obtained by learning through observation. Currently, with fewer operative aneurysm clippings, stricter work-hour regulations, and increased patient safety concerns, novel teaching methods are required for young neurosurgeons. Virtual-reality (VR) models offer the opportunity to either train a specific surgical skill or prepare for an individual surgery. With this study, the authors aimed to compare the spatial orientation between traditional 2D images and 3D VR models in neurosurgical residents or medical students.
\end{abstract}

METHODS Residents and students were each randomly assigned to describe 4 aneurysm cases, which could be either 2D images or 3D VR models. The time to aneurysm detection as well as a spatial anatomical description was assessed via an online questionnaire and compared between the groups. The aneurysm cases were 10 selected patient cases treated at the authors' institution.

RESULTS Overall, the time to aneurysm detection was shorter in the 3D VR model compared to 2D images, with a trend toward statistical significance ( $25.77 \pm 37.26$ vs $45.70 \pm 51.94$ seconds, $p=0.052)$. No significant difference was observed for residents (3D VR $24.47 \pm 40.16$ vs $2 \mathrm{D} 33.52 \pm 56.06$ seconds, $p=0.564$ ), while in students a significantly shorter time to aneurysm detection was measured using 3D VR models $(26.95 \pm 35.39$ vs $59.16 \pm 44.60$ seconds, $p=$ 0.015). No significant differences between the modalities for anatomical and descriptive spatial mistakes were observed. Most participants (90\%) preferred the 3D VR models for aneurysm detection and description, and only 1 participant (5\%) described VR-related side effects such as dizziness or nausea.

CONCLUSIONS VR platforms facilitate aneurysm recognition and understanding of its spatial anatomy, which could make them the preferred method compared to $2 \mathrm{D}$ images in the years to come.

https://thejns.org/doi/abs/10.3171/2021.5.FOCUS21212

KEYWORDS virtual reality; aneurysm surgery; vascular neurosurgery; resident teaching; augmented reality

A NEURYSM surgery is a complex neurosurgical field requiring years of training and a thorough understanding of the 3D neurovascular anatomy. The spatial 3D comprehension of the aneurysm location, dome orientation, and branching vessels has had to be derived from 2D images, a particularly challenging process for young neurosurgeons. ${ }^{1}$ Especially in the current era of frequent endovascular aneurysm treatment, operative aneurysm cases are becoming rarer and often have complex anatomical characteristics, and therefore are not always suitable as teaching cases. Additionally, with the rising costs of operating theater time and the regulations of residents' working hours, hands-on practice and surgical experience are becoming less frequent and residents often lack a sufficient case load. ${ }^{2,3}$ Hence, novel tools are needed to optimize resident teaching and surgical training. Virtual-reality (VR) platforms offer the opportunity to either train a specific surgical skill or to facilitate the surgeon's preparation for a patient-specific case..$^{4-12}$ Some models, including several equipped with haptic feedback to practice procedures, have been successfully used in neurosurgical resident training. ${ }^{4,8,9,13}$ In general, the evi-

ABBREVIATIONS MCA = middle cerebral artery; PICA = posterior inferior cerebellar artery; $\mathrm{VR}=$ virtual reality

SUBMITTED April 1, 2021. ACCEPTED May 13, 2021.

INCLUDE WHEN CITING DOI: 10.3171/2021.5.FOCUS21212.

* J.S. and R.G. contributed equally to this work. 
TABLE 1. Questions in the survey with possible answer options

\begin{tabular}{lc}
\hline \multicolumn{1}{c}{ Question } & Possible Answers \\
\hline 1. What is your gender? & Male, female \\
\hline 2. What is your age? & No. of yrs \\
\hline 3. How many years of neurosurgical practice do you have? & $0,1,2,3,4,5,6,7,8,9,10$ \\
\hline 4. Are you a student or a resident/young neurosurgeon? & Student, resident \\
\hline 5. Case number & No. of seconds \\
\hline 6. Time to detect the aneurysm (seconds) & Saccular, additional bleb, polylobulated \\
\hline 7. Where is the aneurysm located? & Lateral, medial, anterior, posterior, cranial, caudal \\
\hline 8. What is the morphology of the aneurysm? & VR model, 2D images \\
\hline 9. How is the aneurysm dome oriented? & Lt MCA, Rt MCA, ACOM, BA, Lt PICA, Rt PICA, Lt ICA, Rt ICA, PCOM \\
\hline 10. How would you treat the aneurysm? & Yes, no \\
\hline 11. Which modality do you find easier to detect and describe aneurysms? & \\
\hline 12. How do you rate the image quality? & Vood, good, passable, bad, very bad \\
\hline 13. Did you experience any symptoms like nausea, dizziness, double vision, & \\
etc., while using the VR platform? &
\end{tabular}

$\mathrm{ACOM}=$ anterior communicating cerebral artery; $\mathrm{BA}=$ basilar artery; ICA = internal carotid artery; $\mathrm{PCOM}=$ posterior communicating cerebral artery.

dence on objective teaching advantages between 3D VR models and traditional learning methods is scarce. ${ }^{8,9,14}$ SpectoVR is the 3D VR software used in this study and features a fully immersive VR platform allowing for the creation of a patient-specific 3D VR model based on 2D DICOM images. ${ }^{15}$

In this study, we aimed to analyze how quickly neurosurgical residents and medical students comprehend the anatomical characteristics and spatial orientation of different aneurysms when using 3D VR as compared to standard image visualization on a radiology monitor. We hypothesized that with 3D VR it is easier to detect and describe the spatial anatomy of the aneurysm as compared to conventional image visualization.

\section{Methods \\ Study Participants}

The study group consisted of neurosurgery residents (referred to as the doc group) from the Department of Neurosurgery, University Hospital of Basel, and medical students (4th-6th years, referred to as the med group) from the Faculty of Medicine, University of Basel. None of the participants from the doc group had independently performed aneurysmal clipping. The participants from the med group were either interns at our clinic or volunteered to take part in this study; all students passed their neuroanatomy course and examination before participating in the trial. Every participant analyzed 4 aneurysm cases and filled in a web-based questionnaire (Google Forms, Google Inc.) containing demographic questions, questions regarding the anatomical and spatial anatomy of the aneurysm, and questions about image quality and subjective impression of the two methods used (Table 1). The doc group also answered a question concerning their suggested aneurysm treatment (question 10, Table 1), which was omitted in the med group due to a knowledge gap in that group. The aneurysm cases were 10 selected patient-based cases treated at our institution between 2018 and 2020. We prepared a 2D image, either a CT angiogram or an MR angiogram, and a 3D VR data set for each case, resulting in 20 cases (10 per modality). The 20 cases were randomly assigned to the participants using an online randomization tool (randomlists.org), which ensured that the same case was analyzed by more than 1 participant. Participants were able to familiarize themselves with the VR platform using an example case of a middle cerebral artery (MCA) aneurysm, which was not used for the study analysis.

\section{Outcome Variables}

The primary outcome of the study was time to aneurysm detection for the two modalities (3D VR vs 2D). The time was measured in seconds from the moment the image was presented to the participants until the moment they detected the aneurysm. Secondary outcomes were accuracy of describing the anatomical and spatial features of the aneurysm, consisting of side and anatomical location, dome orientation (frontal, posterior, cranial, caudal, medial, or lateral) and morphology (saccular, saccular with a bleb, or polylobulated). All answers were compared between the two modalities for all participants, and for the two groups (doc group and med group) separately. As an exploratory analysis, linear regression was calculated for the time to aneurysm detection and the experience of all participants.

No approval from the local ethics board was needed for this study, but the use of patient images for rendering anonymous 3D VR data sets was registered and approved by the local ethics board.

\section{SpectoVR Software Description}

SpectoVR software renders a fully immersive 3D VR model based on patient-specific DICOM data. The DI$\mathrm{COM}$ images were either based on $\mathrm{CT}$ angiography or digital subtraction angiography images. Threshold settings based on Hounsfield units had to be individually adapted in the program to create a colorful high-contrast 
and user-friendly 3D VR model. The software was written in $\mathrm{C}++$ computer language and developed at the Department of Biomedical Engineering, University of Basel. It was $\mathrm{CE}$ certified as a class I medical device (Diffuse Inc.), and its geometrical and anatomical accuracy has been previously verified. ${ }^{16,17}$ The algorithm used is based on ray casting, in which a ray of light is cast through space, and color and opacity are accumulated along this ray. The visualization is based on a real-time calculation with 180 frames per second, each one corrected for the user's current perspective on the object and the user's manipulation of the object with a controller (scaling, movement in space, interaction with the model), ensuring an immersive viewing experience through a commercial VR headset. For this study, we used HTC Vive glasses and HTC Vive controllers (HTC-Vive, Valve HTC headset, Valve Corp.). This creates a fully immersive VR experience in which the participants are able to interact with the model and move it freely in a virtual operating room (Fig. 1).

\section{Statistical Analysis}

All data collected were imported into an $\mathrm{R}$ database (version 1.3.1093, R Foundation for Statistical Computing) for statistical analysis. Descriptive statistics were conducted for all questions. Comparable contingency statistics was conducted using the Mann-Whitney U-test, Fisher exact test, and chi-square test. A p value $<0.05$ was considered statistically significant.

\section{Results}

\section{Overall Demographics}

Ten participants were included in each group (20 total participants), and each participant analyzed 4 different aneurysms cases in a randomized fashion. This resulted in 80 aneurysm images analyzed in total (40 images per modality). The mean participant age was $27.14 \pm 3.46$ years with a significant difference between the doc group and the med group $(30.25 \pm 1.82$ vs $24.02 \pm 1.00$ years, $p$ $<0.001)$. There were significantly more males in the doc group $(8,80.0 \%)$ compared to the med group $(4,40.0 \%$; $p$ $=0.001$ ). The average neurosurgical experience in the doc group was $4.10 \pm 2.15$ years. When comparing the $2 \mathrm{D}$ and VR groups, the baseline parameters were equally distributed (Table 2). An overview of the aneurysms presented to the participants is shown in Table 3.

\section{Time to Detect and Description of Aneurysms}

Overall time to aneurysm detection was shorter using 3D VR compared to 2D images, with a trend toward statistical significance $(25.77 \pm 37.26$ vs $45.70 \pm 51.94 \mathrm{sec}-$ onds, $\mathrm{p}=0.052$; Fig. 2A). All aneurysms were detected, but in $8(10 \%)$ of the total 80 cases an anatomical mistake occurred. In 3 of the 8 anatomical mistakes, the location of the aneurysm was described incorrectly, and these 3 mistakes occurred in a case involving a posterior inferior cerebellar artery (PICA) aneurysm. The remaining 5 mistakes were made by identifying the wrong side, but the aneurysm location was correct. In total, 3 of the 8 mistakes happened using VR while 5 occurred using 2D images (3D VR $=3[7.5 \%], 2 \mathrm{D}=5$ [12.5\%], $\mathrm{p}=0.709)$. No signifi-

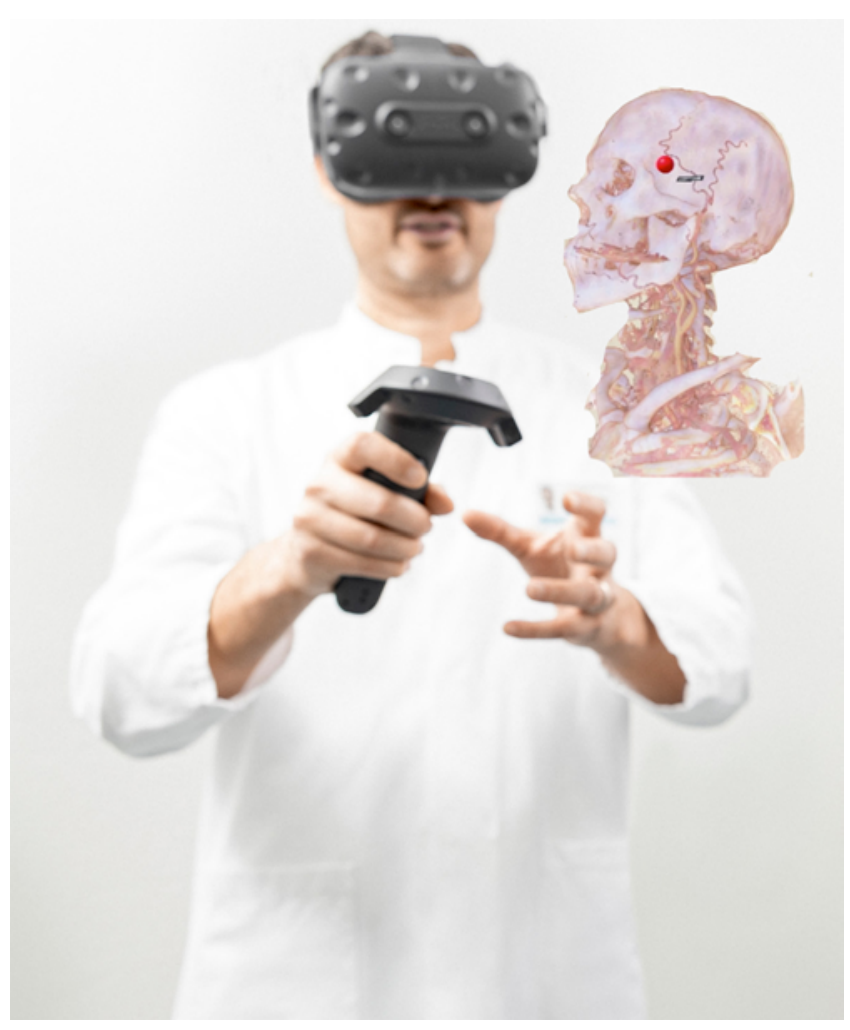

FIG. 1. Participant using SpectoVR to analyze an aneurysm case with a VR headset for a fully immersive experience.

cant differences between the modalities were observed for anatomical (3D VR $=3$ [7.5\%], 2D $=5$ [12.5\%], $\mathrm{p}=0.709$ ) and descriptive (3D VR $=10$ [25.0\%], 2D $=12$ [30.0\%], $\mathrm{p}$ $=0.802$ ) spatial mistakes.

TABLE 2. Baseline parameters in the neurosurgical residents (doc group) and medical students (med group)

\begin{tabular}{lrr}
\hline \multicolumn{1}{c}{ Parameter } & VR Model & \multicolumn{1}{c}{ 2D Images } \\
\hline \multicolumn{1}{l}{ Overall } & \multicolumn{1}{c}{ 40 } & \multicolumn{1}{c}{40} \\
\hline No. of participants & $26.98(3.22)$ & $27.30(3.71)$ \\
\hline Mean age (SD), yrs & $24(60.0)$ & $25(62.5)$ \\
\hline Males (\%) & $1.98(2.48)$ & $2.12(2.66)$ \\
\hline $\begin{array}{l}\text { Mean neurosurgical experience } \\
\text { (SD) yrs }\end{array}$ & &
\end{tabular}

Doc group

\begin{tabular}{|lrr|}
\hline No. of participants & \multicolumn{1}{c}{19} & \multicolumn{1}{c}{21} \\
\hline Mean age (SD), yrs & $30.11(1.29)$ & $30.38(2.22)$ \\
\hline Males (\%) & $16(84.2)$ & $16(76.2)$ \\
\hline $\begin{array}{l}\text { Mean neurosurgical experience } \\
\text { (SD), yrs }\end{array}$ & $4.16(1.92)$ & $4.05(2.38)$ \\
\hline
\end{tabular}

Med group

\begin{tabular}{lrr}
\hline No. of participants & \multicolumn{1}{c}{21} & \multicolumn{1}{c}{19} \\
\hline Mean age (SD), yrs & $24.14(1.01)$ & $23.89(0.99)$ \\
\hline Males (\%) & $8(38.1)$ & $9(47.4)$ \\
\hline $\begin{array}{l}\text { Mean neurosurgical experience } \\
\text { (SD) yrs }\end{array}$ & $0(0.0)$ & $0(0.0)$
\end{tabular}

(SD), yrs 
TABLE 3. Overview of the aneurysm cases used in this study

\begin{tabular}{cccc}
\hline Case & Location & Spatial Orientation & Morphology \\
\hline 1 & ACOM & Frontal, cranial & Saccular w/ extra bleb \\
\hline 2 & Rt MCA & Temporal & Saccular w/ extra bleb \\
\hline 3 & Lt MCA & Temporal & Saccular \\
\hline 4 & Lt PICA & Frontal, cranial & Saccular \\
\hline 5 & Lt MCA & Caudal & Saccular \\
\hline 6 & ACOM & Frontal, cranial & Saccular \\
\hline 7 & ACOM & Frontal & $\begin{array}{c}\text { Saccular w/ 2 extra } \\
\text { blebs (polylobulated) }\end{array}$ \\
\hline 8 & BA & Cranial & Saccular \\
\hline 9 & Rt MCA & Temporal & Saccular \\
\hline 10 & $\begin{array}{c}\text { ACOM, dog-ear } \\
\text { remnant after } \\
\text { previous clipping }\end{array}$ & Lateral & Saccular \\
\hline
\end{tabular}

In the doc group, no significant difference was noted between the two modalities in time to aneurysm detection (3D VR $=24.47 \pm 40.16$ vs $2 \mathrm{D}=33.52 \pm 56.06$ seconds, $\mathrm{p}$ $=0.564$; Fig. 2B). No significant difference was observed between the modalities for anatomical mistakes (3D VR = 3 [15.8\%], $2 \mathrm{D}=1$ [4.8\%], $\mathrm{p}=0.527)$ or for incorrect spatial description of the aneurysm (3D VR $=4$ [21.1\%], 2D $=3$ [14.3\%], $\mathrm{p}=0.884)$.

In the med group, the time to aneurysm detection reached statistical significance in favor of the 3D VR modality $(26.95 \pm 35.39$ vs $59.16 \pm 44.60$ seconds, $\mathrm{p}=0.015$; Fig. 2C). No incorrect anatomical description occurred using 3D VR models (3D VR $=0[0.0 \%], 2 \mathrm{D}=4[21.1 \%], \mathrm{p}$
$=0.091)$. Incorrect spatial orientation was observed more often using 2D images, however, no statistical significance was reached (3D VR $=6$ [28.6\%], 2D $=9$ [47.4\%], $\mathrm{p}=$ 0.369).

Linear regression analysis showed that for the 2D modality, experience has a significant correlation with the time required to detect the aneurysm, while in $3 \mathrm{D}$ VR models no such correlation was detected (Fig. 3).

\section{Subjective Impression}

Two participants (10\%, 1 from each group) found the 2D images easier to identify, describe morphology, and select a treatment method for the aneurysm, while the remaining participants $(90 \%)$ preferred 3D VR. One participant (5\%) in the doc group described dizziness and nausea while using the 3D VR platform. The overall image quality of the 3D VR models was reported as good or very good in $75.6 \%$; an overview of the image quality ratings is shown in Table 4.

\section{Discussion}

In this prospective randomized study, we observed that the time to aneurysm detection showed a shorter duration when using 3D VR models compared to 2D images. Medical students, who lack experience in cerebrovascular anatomy, may particularly benefit from the 3D VR modality, with significantly shorter time to aneurysm detection. Most participants (90\%) found it easier using the VR model to detect and describe the aneurysm and its spatial anatomy. Only 1 participant (5\%) described VR-related side effects such as nausea and dizziness. This is one of the few studies that has analyzed objective teaching and
A

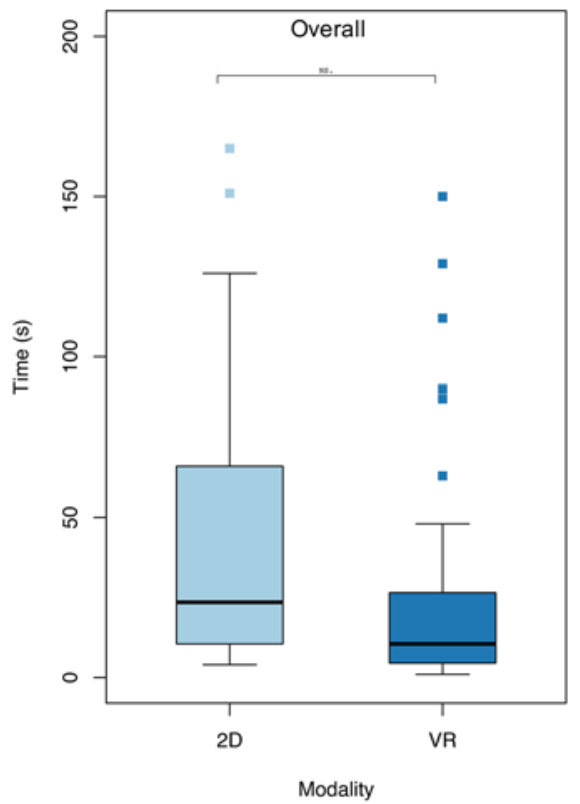

B

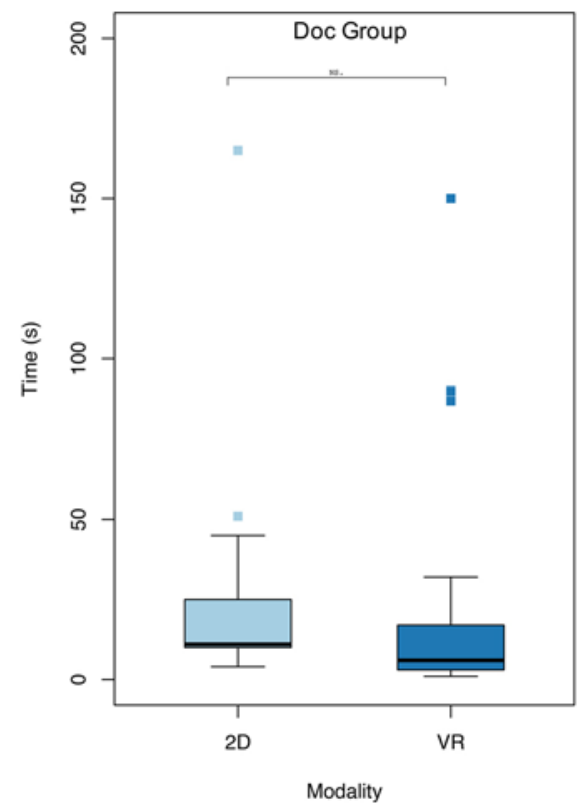

C Time to detect Aneurysm

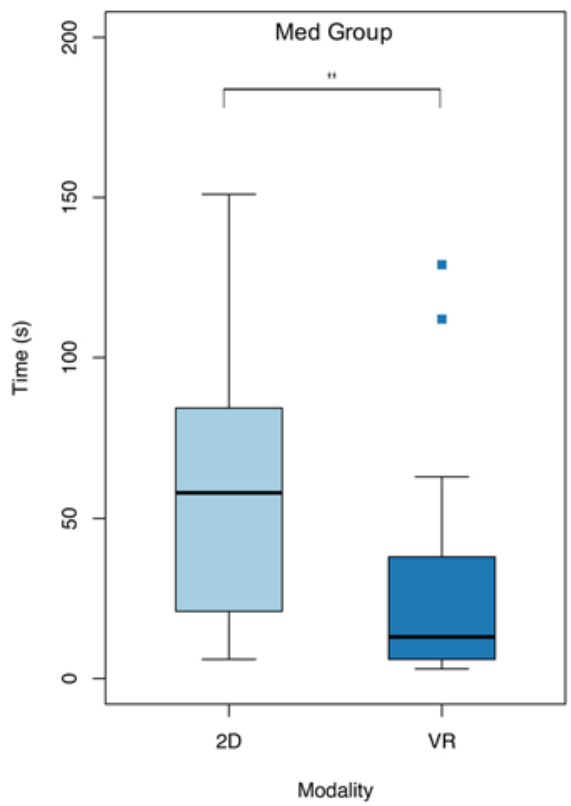

FIG. 2. Boxplots showing the time to aneurysm detection using 3D VR models or $2 \mathrm{D}$ images in all participants (A), the doc group (B), and the med group (C). ${ }^{* *} p<0.05$. NS. = nonsignificant. 

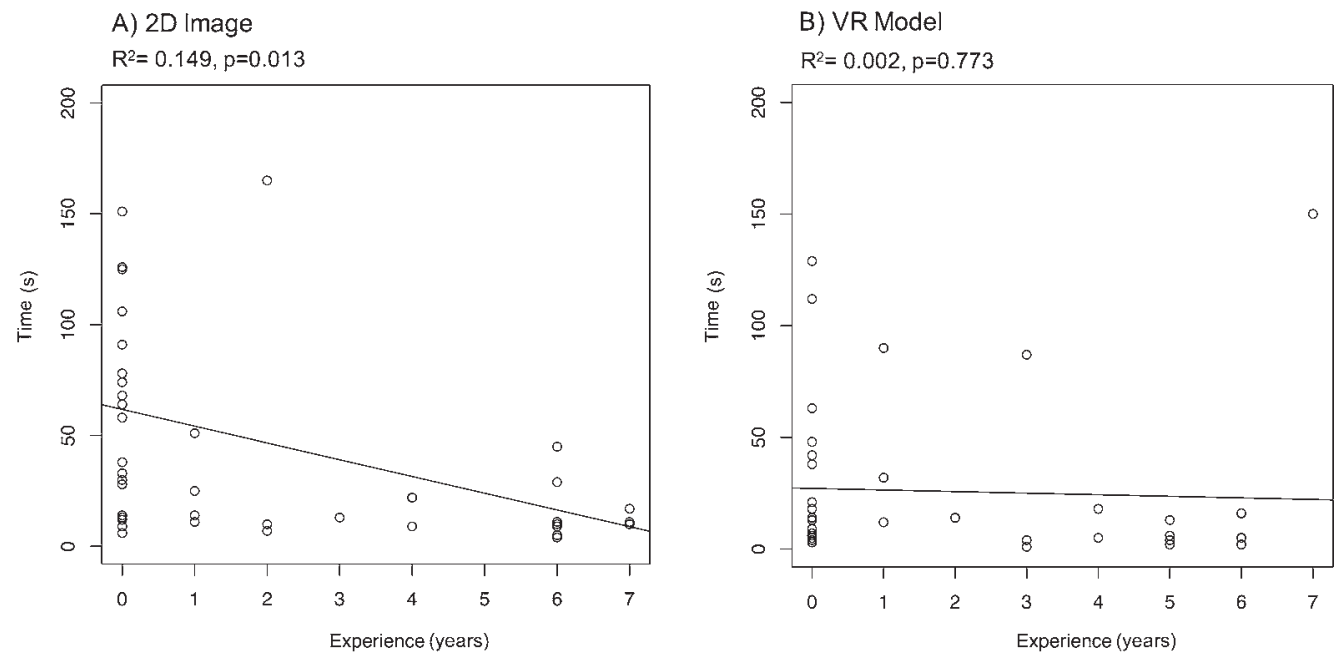

FIG. 3. Linear regression model for time and experience using a 2D image (A) and 3D VR model (B).

training data between 3D VR and 2D images in medical students and residents, and to our knowledge the first one of this nature in neurosurgery. ${ }^{9,14}$

VR as a surgical teaching and training tool has become more common in the last decades due to technical progress, limited patient exposure for medical students and residents, increased patient safety concerns, and the high cost of operating time. ${ }^{2,18}$ Our results show that medical students, who lack the experience in analyzing 2D images and creating a mental 3D model thereof, particularly benefit from using 3D VR. VR appears to be able to compensate for years of experience, required to successfully analyze 2D images. Similar experiences were reported, as VR can allow an enhanced learning experience accelerating the learning curve for spatial anatomy, which traditionally was obtained by years of learning through observation. ${ }^{2,18}$ However, after several training units in different VR platforms, a plateauing of the learning curve was observed. This indicates that VR might be able to accelerate the learning curve of novices in the beginning, but later on more traditional and supervised training is probably required. ${ }^{19-21}$ However, in our experience SpectoVR also enhances surgical preparation at a later stage in training by analyzing the spatial anatomy of the patient-specific case. Based on the senior authors' experience, it is very helpful to study the anatomy and vessels adjacent to the aneurysm for surgical planning. SpectoVR models can assist in determining how to best approach the aneurysm and the clip placement angle and create a so-called "déjà-vu effect" intraoperatively, facilitating the surgeon's orientation. ${ }^{5}$ These factors were not measured in this project but are subject to further studies. Use of SpectoVR is standard in our institution to prepare for cerebrovascular cases. Because it can access imaging directly from the PACS radiology system and does not require any preprocessing, SpectoVR can be used seamlessly for everyday cases. Moreover, SpectoVR has also been successfully used in complex spine surgery to plan the surgical approach and pedicle screw placement. ${ }^{15,22}$

Several different VR platforms for teaching and training have already been used and described in neurosurgery.,7,-9,
${ }^{12,23-27}$ Most of these were designed to practice a specific procedure or plan a surgical approach and were descriptive studies without describing a comparison to conventional 2D methods. ${ }^{4,5,7,8,14,23,25}$ Additionally, VR models have also been described for teaching and studying neuroanatomy, but no benefit was observed concerning knowledge retention compared to conventional 2D images..$^{14}$ Only 1 study (Chugh et al. ${ }^{9}$ ) conducted a randomized trial comparing VR to conventional 2D methods. Chugh et al. compared the operative time used for clip placement between a group preparing with the help of a VR platform for aneurysm

TABLE 4. Image quality of the VR model and 2D images rated by the participants

\begin{tabular}{cccc}
\hline Quality & VR Model (\%) & 2D Images (\%) & p Value \\
\hline Overall & & & 0.069 \\
\hline Very good & $12(32.4)$ & $4(10.5)$ & \\
\hline Good & $16(43.2)$ & $16(42.1)$ & \\
\hline Passable & $7(18.9)$ & $15(39.5)$ & \\
\hline Bad & $2(5.4)$ & $3(7.9)$ & \\
\hline Very bad & $0(0.0)$ & $0(0.0)$ & \\
\hline Doc group & & & \\
\hline Very good & $5(31.2)$ & $4(21.1)$ & \\
\hline Good & $7(43.8)$ & $6(31.6)$ & \\
\hline Passable & $3(18.8)$ & $8(42.1)$ & \\
\hline Bad & $1(6.2)$ & $1(5.3)$ & \\
\hline Very bad & $0(0.0)$ & $0(0.0)$ & \\
\hline Med group & & & \\
\hline Very good & $7(33.3)$ & $0(0.0)$ & \\
\hline Good & $9(42.9)$ & $10(52.6)$ & \\
\hline Passable & $4(19.0)$ & $7(36.8)$ & \\
\hline Bad & $1(4.8)$ & $2(10.5)$ & \\
\hline Very bad & $0(0.0)$ & $0(0.0)$ & \\
\hline
\end{tabular}

Boldface type indicates statistical significance. 
clipping, to a group using standard preparation techniques. The overall operative time was significantly shorter in the group preparing by using the VR platform, and fewer clip placement attempts were needed until final occlusion of the aneurysm was reached in the VR group. ${ }^{9}$ These results are congruent with the results from this study and indicate that VR platforms assist neurosurgeons in teaching and surgical training of cerebrovascular cases. In the study by Chugh et al., Surgical Planner (SRP; Surgical Theater LLC) was used, which is an FDA-approved VR platform. SRP allows 3D patient-specific planning and rehearsing of a procedure but lacks a fully immersive feature and haptic feedback. ${ }^{9}$ The option to select different surgical instruments and perform clip placement is one of the main differences between SRP and SpectoVR, which currently only offers the possibility to perform virtual craniotomies. SpectoVR, however, does offer a fully immersive experience allowing the participant to interact with the model in a virtual operating theater, which is still helpful for surgical preparation and profound understanding of the spatial anatomy necessary to perform successful clip ligation of the aneurysm. Two other well-known VR platforms used in cerebrovascular surgery are the Dextroscope (Volume Interactions, Pte, Ltd.) and ImmersiveTouch platforms. ${ }^{4,6,7,28}$ The ImmersiveTouch platform allows a fully immersive experience with haptic feedback but is not described as patient-specific; it serves the purpose of skill training more than surgical preparation for a specific case., ${ }^{4,28}$ Alaraj et al. described the experience of residents training for MCA aneurysm clipping using the ImmersiveTouch platform. ${ }^{4}$ Most residents $(64 \%)$ found that VR training of aneurysm clipping was helpful and improved their anatomical understanding of the aneurysm. ${ }^{4}$ In contrast, the Dextroscope is mostly described as a 3D VR surgical preparation but not skilltraining platform. ${ }^{1,7,29,30}$ It allows patient-specific $3 \mathrm{D}$ visualization of the anatomy but lacks haptic feedback and fully immersive surroundings.

A possible downside of VR-based teaching and training models is the so-called "simulator sickness," which is caused by a visual-vestibular interaction. Symptoms are similar to motion sickness and mostly occur in firsttime users, while an improvement over time has been described. ${ }^{31-33}$ In this study, only 1 participant (5\%) described symptoms of simulator sickness, which indicates that such symptoms, at least in this cohort, are even more rare than previously described. ${ }^{34}$

\section{Study Limitations}

Limitations of the study are its small size and singlecenter experience. While we observed an overall tendency that VR is superior to 2D images, the study was underpowered to find a statistically significant difference. Additionally, SpectoVR is an academic VR platform, which has not been validated in comparison to commercially available VR platforms. Furthermore, no conclusion can be drawn to any possible surgical performance after preparation with SpectoVR as this study solely focused on the anatomical description. However, to our knowledge this is only the second randomized trial comparing 3D VR models to conventional 2D images in aneurysm surgery, and neurosurgery in general.

\section{Conclusions}

VR platforms facilitate aneurysm recognition and understanding of its spatial anatomy, which could make them the preferred method compared to $2 \mathrm{D}$ images in the years to come. A significantly shorter time to detect the aneurysm was observed in the 3D VR group, and participants with little experience especially benefit from 3D VR models. In the future, VR platforms could play an essential role in teaching and training, but further larger trials are needed to prove its standard use in neurosurgery.

\section{Acknowledgments}

Dr. Croci has been financially supported by a grant from the Swiss Academy for Medical Sciences (SAMW) and has received a grant from the Nora van Meeuwen-Haefliger Foundation.

\section{References}

1. Ferroli P, Tringali G, Acerbi F, Schiariti M, Broggi M, Aquino D, Broggi G. Advanced 3-dimensional planning in neurosurgery. Neurosurgery. 2013;72(suppl 1):54-62.

2. Alaraj A, Lemole MG, Finkle JH, Yudkowsky R, Wallace A, Luciano C, et al. Virtual reality training in neurosurgery: review of current status and future applications. Surg Neurol Int. 2011;2(1):52.

3. Bridges M, Diamond DL. The financial impact of teaching surgical residents in the operating room. Am J Surg. 1999; 177(1):28-32.

4. Alaraj A, Luciano CJ, Bailey DP, Elsenousi A, Roitberg BZ, Bernardo A, et al. Virtual reality cerebral aneurysm clipping simulation with real-time haptic feedback. Neurosurgery. 2015;11(1)(suppl 2):52-58.

5. Kockro RA, Serra L, Tseng-Tsai Y, Chan C, Yih-Yian S, Gim-Guan C, et al. Planning and simulation of neurosurgery in a virtual reality environment. Neurosurgery. 2000;46(1): 118-137.

6. Kockro RA, Stadie A, Schwandt E, Reisch R, Charalampaki $\mathrm{C}, \mathrm{Ng}$ I, et al. A collaborative virtual reality environment for neurosurgical planning and training. Neurosurgery. 2007; 61(5)(suppl 2):379-391.

7. Kockro RA, Killeen T, Ayyad A, Glaser M, Stadie A, Reisch $\mathrm{R}$, et al. Aneurysm surgery with preoperative three-dimensional planning in a virtual reality environment: technique and outcome analysis. World Neurosurg. 2016;96:489-499.

8. Banerjee PP, Luciano CJ, Lemole GM Jr, Charbel FT, Oh MY. Accuracy of ventriculostomy catheter placement using a head- and hand-tracked high-resolution virtual reality simulator with haptic feedback. J Neurosurg. 2007;107(3):515-521.

9. Chugh AJ, Pace JR, Singer J, Tatsuoka C, Hoffer A, Selman WR, Bambakidis NC. Use of a surgical rehearsal platform and improvement in aneurysm clipping measures: results of a prospective, randomized trial. J Neurosurg. 2017;126(3):838-844.

10. Bambakidis NC, Selman WR, Sloan AE. Surgical rehearsal platform: potential uses in microsurgery. Neurosurgery. 2013; 73(suppl 1):122-126.

11. Fiani B, De Stefano F, Kondilis A, Covarrubias C, Reier L, Sarhadi K. Virtual reality in neurosurgery: "Can you see it?"-a review of the current applications and future potential. World Neurosurg. 2020;141:291-298.

12. Baby B, Singh R, Suri A, Dhanakshirur RR, Chakraborty A, Kumar S, et al. A review of virtual reality simulators for neuroendoscopy. Neurosurg Rev. 2020;43(5):1255-1272.

13. Licci M, Thieringer FM, Guzman R, Soleman J. Development and validation of a synthetic 3D-printed simulator for training in neuroendoscopic ventricular lesion removal. $\mathrm{Neu}$ rosurg Focus. 2020;48(3):E18. 
14. Stepan K, Zeiger J, Hanchuk S, Del Signore A, Shrivastava $\mathrm{R}$, Govindaraj S, Iloreta A. Immersive virtual reality as a teaching tool for neuroanatomy. Int Forum Allergy Rhinol. 2017;7(10):1006-1013.

15. Croci DM, Guzman R, Netzer C, Mariani L, Schaeren S, Cattin PC, Jost GF. Novel patient-specific 3D-virtual reality visualisation software (SpectoVR) for the planning of spine surgery: a case series of eight patients. BMJ Innov. 2020;6(4): 215-219.

16. Maloca PM, Faludi B, Zelechowski M, Jud C, Vollmar T, Hug S, et al. Validation of virtual reality orbitometry bridges digital and physical worlds. Sci Rep. 2020;10(1):11815.

17. Maloca PM, de Carvalho JER, Heeren T, Hasler PW, Mushtaq F, Mon-Williams M, et al. High-performance virtual reality volume rendering of original optical coherence tomography point-cloud data enhanced with real-time ray casting. Transl Vis Sci Technol. 2018;7(4):2.

18. Bernardo A. Virtual reality and simulation in neurosurgical training. World Neurosurg. 2017;106:1015-1029.

19. Selvander M, Åsman P. Virtual reality cataract surgery training: learning curves and concurrent validity. Acta Ophthalmol. 2012;90(5):412-417.

20. Brown C, Robinson D, Egan R, Hopkins L, Abdelrahman T, Powell A, et al. Prospective cohort study of haptic virtual reality laparoscopic appendicectomy learning curve trajectory. J Laparoendosc Adv Surg Tech A. 2019;29(9):1128-1134.

21. Andersen SAW, Konge L, Cayé-Thomasen P, Sørensen MS. Learning curves of virtual mastoidectomy in distributed and massed practice. JAMA Otolaryngol Head Neck Surg. 2015; 141(10):913-918.

22. Zoller EI, Faludi B, Gerig N, Jost GF, Cattin PC, Rauter G. Force quantification and simulation of pedicle screw tract palpation using direct visuo-haptic volume rendering. Int $J$ CARS. 2020;15(11):1797-1805.

23. Lemole M, Banerjee PP, Luciano C, Charbel F, Oh M. Virtual ventriculostomy with 'shifted ventricle': neurosurgery resident surgical skill assessment using a high-fidelity haptic/graphic virtual reality simulator. Neurol Res. 2009;31(4):430-431.

24. Schirmer CM, Elder JB, Roitberg B, Lobel DA. Virtual reality-based simulation training for ventriculostomy: an evidence-based approach. Neurosurgery. 2013;73(suppl 1): 66-73.

25. Luciano CJ, Banerjee PP, Sorenson JM, Foley KT, Ansari SA, Rizzi S, et al. Percutaneous spinal fixation simulation with virtual reality and haptics. Neurosurgery. 2013;72(suppl 1):89-96.

26. Gmeiner M, Dirnberger J, Fenz W, Gollwitzer M, Wurm G, Trenkler J, Gruber A. Virtual cerebral aneurysm clipping with real-time haptic force feedback in neurosurgical education. World Neurosurg. 2018;112:e313-e323.

27. Tomlinson SB, Hendricks BK, Cohen-Gadol A. Immersive three-dimensional modeling and virtual reality for enhanced visualization of operative neurosurgical anatomy. World Neurosurg. 2019;131:313-320.
28. Lemole GM Jr, Banerjee PP, Luciano C, Neckrysh S, Charbel FT. Virtual reality in neurosurgical education: part-task ventriculostomy simulation with dynamic visual and haptic feedback. Neurosurgery. 2007;61(1):142-149.

29. Du ZY, Gao X, Zhang XL, Wang ZQ, Tang WJ. Preoperative evaluation of neurovascular relationships for microvascular decompression in the cerebellopontine angle in a virtual reality environment. J Neurosurg. 2010;113(3):479-485.

30. Ng I, Hwang PYK, Kumar D, Lee CK, Kockro RA, Sitoh YY. Surgical planning for microsurgical excision of cerebral arterio-venous malformations using virtual reality technology. Acta Neurochir (Wien). 2009;151(5):453-463.

31. Kennedy RS, Lane NE, Berbaum KS, Lilienthal MG. Simulator Sickness Questionnaire: An enhanced method for quantifying simulator sickness. Int J Aviat Psychol. 1993;3(3): 203-220.

32. Kim HK, Park J, Choi Y, Choe M. Virtual reality sickness questionnaire (VRSQ): motion sickness measurement index in a virtual reality environment. Appl Ergon. 2018;69:66-73.

33. Sharples S, Cobb S, Moody A, Wilson JR. Virtual reality induced symptoms and effects (VRISE): comparison of head mounted display (HMD), desktop and projection display systems. Displays. 2008;29(2):58-69.

34. Matas NA, Nettelbeck T, Burns NR. Dropout during a driving simulator study: a survival analysis. J Safety Res. 2015; 55:159-169.

\section{Disclosures}

Philippe Cattin is a cofounder of the company Diffuse Inc., which developed the SpectoVR software.

\section{Author Contributions}

Conception and design: Greuter, Croci, Soleman, Guzman. Acquisition of data: Greuter, De Rosa. Analysis and interpretation of data: Greuter. Drafting the article: Greuter, De Rosa. Critically revising the article: Greuter, Soleman, Guzman. Reviewed submitted version of manuscript: all authors. Approved the final version of the manuscript on behalf of all authors: Greuter. Statistical analysis: Greuter. Administrative/technical/material support: Cattin. Study supervision: Soleman, Guzman.

\section{Correspondence}

Ladina Greuter: University Hospital of Basel, Switzerland. ladina.greuter@usb.ch. 